\title{
Adaptação e Avaliação de uma Intervenção Cognitivo-Comportamental para Meninos Vítimas de Violência Sexual
}

\author{
Adaptation and Evaluation of a Cognitive-Behavioral Intervention \\ for Boys who have Been Sexually Abused
}

\author{
Jean Von Hohendorff ${ }^{*},{ }^{a}$, Roberta Salvador-Silva ${ }^{b}$, Rosiane de Andrade ${ }^{c}$, \\ Luísa Fernanda Habigzang $^{b} \&$ Silvia Helena Koller ${ }^{a}$ \\ ${ }^{a}$ Universidade Federal do Rio Grande do Sul, Porto Alegre, Rio Grande do Sul, Brasil, \\ ${ }^{b}$ Pontifíicia Universidade Católica do Rio Grande do Sul, Porto Alegre, Rio Grande do Sul, Brasil \\ \& Instituto de Desenvolvimento Global, Porto Alegre, Rio Grande do Sul, Brasil
}

\begin{abstract}
Resumo
Objetivou-se adaptar, aplicar e avaliar um modelo de intervenção cognitivo-comportamental para meninos vítimas de Violência Sexual (VS). Participaram três meninos com idades entre oito e 16 anos, vítimas de VS, que foram avaliados antes e após a intervenção com instrumentos psicológicos acerca de transtornos disruptivos, de humor e de ansiedade. A aplicação do modelo foi avaliada por juízes que analisaram os relatos das sessões terapêuticas por meio de seis indicadores. Os resultados indicaram variabilidade no número de sintomas de cada participante. A avaliação da aplicação revelou a adequação do modelo quanto a dois indicadores (Aliança Terapêutica e Autorrevelação), bem como reajustes necessários no modelo adaptado. Esses resultados se constituem como evidências iniciais sobre a ação psicoterapêutica do modelo adaptado.

Palavras-chave: Violência sexual, meninos, terapia cognitivo-comportamental.
\end{abstract}

\begin{abstract}
This work aimed to adapt, apply and assess a cognitive-behavioral intervention model for boys who have been victims of sexual violence (SV). Three boys between eight and 16 years old who had been victims of SV were evaluated through psychological instruments before and after the intervention in terms of humor, anxiety and disruptive symptoms. Three independent judges analyzed reports of therapeutic sessions in order to evaluate the application of the adapted model through six indicators. Results showed variability in the number of symptoms for each boy. The evaluation indicated adequacy of two indicators (Therapeutic Alliance and Self-Disclosure), but the need of some readjustments in the adapted model. These findings provide initial proof about the effectiveness of the adapted therapeutic model.
\end{abstract}

Keywords: Sexual violence, boys, cognitive-behavioral therapy.

O número de pesquisas sobre a violência sexual (VS) aumentou consideravelmente nas últimas décadas, sendo conduzidas majoritariamente com vítimas femininas, dificultando a generalização de seus resultados para vítimas masculinas (Maikovich-Fong \& Jaffee, 2010). A escassez de publicações sobre VS masculina pode estar atrelada ao menor número de casos notificados nessa população

\footnotetext{
* Endereço para correspondência: Universidade Federal do Rio Grande do Sul, Rua Ramiro Barcelos, 2600, Sala 104, Porto Alegre, RS, Brasil 90035-003. E-mail: jhohendorff@gmail.com

O artigo faz parte da dissertação intitulada "Adaptação e avaliação de uma intervenção cognitivo-comportamental para meninos vítimas de violência sexual", de autoria do primeiro autor e orientação da quarta e quinta autoras. Os autores agradecem à Dra. Maria Clara Pinheiro de Paula Couto pela ajuda com a análise de dados deste estudo.
}

(Hohendorff, Habigzang, \& Koller, 2012). Essa realidade pode ser explicada pela dificuldade que meninos vítimas possuem em relatar a violência ocorrida (Sigurdardottir, Halldorsdottir, \& Bender, 2012; Weiss, 2010), devido, em parte, a fatores culturais, como a maior visibilidade da VS feminina (Pinto, 2005).

Embora as consequências da VS contra meninos ainda sejam pouco investigadas, estudos indicam que essa forma de violência repercute em prejuízos para o desenvolvimento (Alaggia, 2005; Holmes \& Slap, 1998; Lisak, 1994; Maniglio, 2009; O’Leary, 2009; Pires, 2007). Dentre eles, o transtorno de estresse pós-traumático (TEPT) é indicado como um dos transtornos mais comuns entre vítimas, inclusive masculinas (O’Leary, 2009). Além disso, dúvidas quanto à sua masculinidade e orientação sexual são consequências da VS masculina (Alaggia, 2005; Lisak, 1994), 
bem como a presença de transtornos disruptivos (Holmes \& Slap, 1998). Embora não haja consenso na literatura, acredita-se que sintomas de transtornos disruptivos, tais como o desafiador-opositivo e o de conduta, sejam mais frequentes em meninos vítimas (Maniglio, 2009; Pires, 2007). Em alguns casos, as vítimas podem reproduzir a VS que sofreram e, dessa forma, cometer atos abusivos contra os pares (Holmes \& Slap, 1998).

Diante das consequências da VS para as vítimas, o desenvolvimento e avaliação de intervenções para essa população são necessários. A Terapia Cognitivo-Comportamental (TCC) vem sendo utilizada com crianças e adolescentes vítimas de VS e obtendo evidências de efetividade em estudos internacionais (Deblinger, Mannarino, Cohen, Runyon, \& Steer, 2011; Hetzel-Riggin, Brausch, \& Montgomery, 2007) e nacionais (Habigzang et al., 2009; Lucânia, Valério, Barison, \& Miyazaki, 2009). Os estudos nacionais, no entanto, foram realizados apenas com meninas. Estudos específicos com vítimas masculinas são escassos em nível nacional (Hohendorff, Bavaresco, Habigzang, \& Koller, 2012). Diante disso, o objetivo deste estudo foi adaptar, aplicar e avaliar uma intervenção cognitivo-comportamental (CC) para meninos vítimas de VS, tendo em vista a lacuna nas pesquisas brasileiras sobre essa temática.

\section{Método}

Foi realizado um estudo de casos múltiplos (Yin, 2003/2005) que consiste em desenvolver conhecimento detalhado sobre pequeno número de casos relacionados.

\section{Participantes}

Participaram do estudo três meninos (oito, 10 e 16 anos) vítimas de VS. Os participantes foram encaminhados pelos Conselhos Tutelares (CT) e atendidos em dois centros de atendimento a vítimas de VS da região metropolitana de Porto Alegre. Os critérios de inclusão foram: presença de, no mínimo, um episódio de VS intra ou extrafamiliar, sexo masculino e idade entre oito e 16 anos. Os critérios de exclusão foram: presença de sintomas psicóticos e/ou retardo mental, sendo que nenhum dos participantes encaminhados possuía tais características. A seguir é apresentado um breve relato com as principais informações de cada caso, as quais são complementadas pelas informações da Tabela 1.

Tabela 1

Informações sobre Violência Sexual dos Participantes

\begin{tabular}{|c|c|c|c|}
\hline Informações dos participantes & Participante A & Participante B & Participante $\mathrm{C}$ \\
\hline Idade no início da intervenção & 16 & 08 & 10 \\
\hline Idade de início da VS & 08 & 08 & 09 \\
\hline Idade quando revelou VS & 16 & 08 & 10 \\
\hline Agressor & Familiar paterno & Irmão & Vizinho \\
\hline Número episódios & Múltiplos & Múltiplos & Múltiplos \\
\hline Forma de VS & $\begin{array}{l}\text { Contato físico } \\
\text { sem penetração }\end{array}$ & $\begin{array}{l}\text { Contato físico } \\
\text { sem penetração }\end{array}$ & $\begin{array}{l}\text { Sem contato } \\
\text { físico }\end{array}$ \\
\hline Presença de ameaças & Não & Não & Sim \\
\hline Pessoa a quem revelou & Conselheira Tutelar & Mãe & Avó \\
\hline Credibilidade na revelação & Sim & Sim & Sim \\
\hline Medidas adotadas após a revelação & $\begin{array}{c}\text { Encaminhamento } \\
\text { para tratamento }\end{array}$ & $\begin{array}{l}\text { Participante contou para } \\
\text { uma segunda pessoa (tia) } \\
\text { que notificou a situação }\end{array}$ & Notificação \\
\hline Acolhimento & Sim & Não & Não \\
\hline Responsabilização criminal do agressor & Não & Não & Não \\
\hline Tempo de espera por atendimento psicológico & Imediato & Imediato & Um mês \\
\hline
\end{tabular}

O participante A, 16 anos, foi encaminhado para avaliação psicológica após notificação efetuada por uma tia alegando que o menino estaria cometendo VS contra o irmão (oito anos). O participante confirmou a ocorrência de
VS contra o irmão e relatou que também havia sido vítima, justificando sua inclusão neste estudo. Ele foi encaminhado para acolhimento institucional como medida protetiva referente à VS perpetrada contra o irmão. O participante 
B, oito anos, foi encaminhado para avaliação psicológica após notificação efetuada pela tia, a quem o menino relatou a VS perpetrada pelo irmão de 16 anos (participante A). $\mathrm{O}$ participante $\mathrm{C}, 10$ anos, foi encaminhado para avaliação psicológica após notificação realizada por sua avó, a qual possuía a guarda do menino. Mesmo após a notificação, o agressor (16 anos), que era amigo da vítima e brincavam juntos, permaneceu morando em frente à casa do menino, sendo que não houve mais contato, ou seja, não brincaram mais juntos.

\section{Procedimentos}

O projeto de pesquisa foi aprovado pelo Comitê de Ética do Hospital Materno-Infantil Presidente Vargas, de Porto Alegre, conforme o documento $n^{\circ} 36 / 10$, e seguiu todas as diretrizes éticas vigentes para a realização de estudos com seres humanos. Após isso, deu-se início aos procedimentos de adaptação do modelo, avaliação dos participantes e aplicação do modelo adaptado e avaliação desta aplicação.

Adaptação do Modelo. O modelo de intervenção foi adaptado a partir do modelo de grupoterapia cognitivo-comportamental para meninas vítimas de VS (consultar Habigzang et al., 2009). Esse consiste em 16 sessões semanais, com duração média de uma hora e trinta minutos, divididas em três etapas - Psicoeducação e reestruturação cognitiva, Treino de Inoculação do Estresse (TIE) e Prevenção à Recaída - conforme as técnicas empregadas. A adaptação do modelo compreendeu cinco etapas (consultar Hohendorff, Habigzang, \& Koller, 2013):

1. Reunião com a equipe responsável pela aplicação do modelo utilizado com meninas (Habigzang et al., 2009), com o objetivo de discutir possíveis adaptações à aplicação em meninos;

2. Reunião com um psicólogo que possuía experiência na condução de pesquisas com meninos vítimas de VS, visando a verificar a adequação das adaptações discutidas na etapa anterior;

3. Confirmação, com base na literatura científica, das adaptações propostas nas etapas anteriores;

4. Nova reunião com a equipe responsável pela aplicação do modelo utilizado com meninas (Habigzang et al., 2009) para discussão final das adaptações;

5. Realização de um estudo piloto com um menino vítima de VS, no qual se buscou verificar, na prática, a adequação das adaptações propostas. Para tal, um cuidadoso processo foi realizado, levando em conta os princípios éticos quanto à proteção dos direitos, bem estar e dignidade dos participantes na pesquisa. Após estas etapas, deu-se origem ao modelo de intervenção cognitivo-comportamental adaptado.

$\mathrm{O}$ modelo foi adaptado quanto ao seu formato, passando a ser individual, tendo em vista o número escasso de casos notificados de VS contra meninos (Hohendorff, Habigzang, \& Koller, 2012), bem como a experiência dos autores na condução de intervenções com crianças e adolescentes vítimas de VS, a qual confirmava os dados da literatura. Além disso, três sessões da etapa de psicoeducação e três sessões na etapa de prevenção à recaída foram adaptadas. As sessões do TIE permaneceram iguais. As adaptações das sessões de Psicoeducação consistiram na adequação de dispositivos utilizados no formato de grupo para o formato individual, bem como a substituição do vídeo sobre VS utilizado. Para a psicoeducação quanto à VS, foi produzido um documentário específico sobre VS masculina (consultar Hohendorff, Habigzang, Rodrigues, \& Koller, 2012). Nas sessões de prevenção à recaída, foram incluídas discussões sobre identidade de gênero e orientação sexual, além de adaptações de alguns dispositivos para o formato individual.

Avaliação dos Participantes e Aplicação do Modelo Adaptado. A avaliação dos participantes e a aplicação do modelo foi realizada por três pesquisadores distintos: um psicólogo e duas concluintes do curso de graduação em Psicologia com experiência em TCC. Cada um dos participantes foi atendido por um(a) pesquisador(a) diferente com o intuito de diminuir possíveis vieses nos resultados relacionados a quem aplicaria o modelo. Todos os envolvidos com a aplicação do modelo participaram de um programa de capacitação, com carga horária de 50 horas (Habigzang, Damásio, Hohendorff, \& Koller, 2011). Supervisões semanais durante a aplicação do modelo foram realizadas com os psicoterapeutas/pesquisadores.

Os participantes da pesquisa foram avaliados antes da aplicação do modelo adaptado, em entrevistas individuais, pelo(a) mesmo(a) profissional que conduziu a intervenção para facilitar a vinculação terapêutica. A avaliação foi composta por três encontros semanais com duração de uma hora que teve como objetivo avaliar sintomas de depressão, estresse, ansiedade, TEPT, Dependência/Abuso de substância, TDAH, Transtorno de Conduta (TC) e Transtorno Desafiador-Opositivo (TDO), além de atribuições e percepções relacionadas à VS. A ordem de aplicação dos instrumentos foi aleatória para evitar o efeito de ordem nos resultados. Ao final da aplicação total do modelo, os instrumentos, com exceção da entrevista semi-estruturada inicial, foram reaplicados por outro membro da equipe, que não o responsável pela condução da intervenção, com o objetivo de evitar possíveis vieses relacionados à desejabilidade social.

Avaliação da Aplicação do Modelo Adaptado. Após o término de cada uma das sessões, foram feitos relatos escritos detalhados, visando à avaliação que ocorreu após as 16 sessões do modelo adaptado. Essa avaliação foi realizada por juízes independentes com experiência na condução de pesquisas e intervenções psicoterápicas com vítimas de VS por meio de uma ficha de avaliação dos relatos da intervenção (ver seção Instrumentos). Optou-se por não gravar os atendimentos, uma vez que esse recurso poderia inibir os participantes. 
Hohendorff, J. V., Salvador-Silva, R., Andrade, R., Habigzang, L. F. \& Koller, S. H. (2014). Adaptação e Avaliação de uma Intervenção Cognitivo-Comportamental para Meninos Vítimas de Violência Sexual.

\section{Instrumentos}

Entrevista Semi-Estruturada Inicial. Na primeira parte objetivou-se estabelecer vínculo terapêutico com os participantes. A segunda parte foi baseada em uma entrevista publicada pelo The Metropolitan Toronto Special Committee on Child Abuse (1995), traduzida para o português e adaptada por Kristensen (1996), na qual o objetivo foi obter o relato dos participantes em relação à VS.

Escala de Atribuições e Percepções de Crianças (CAPS; Mannarino, Cohen, \& Berman, 1994, traduzida por Habigzang, 2006). Escala que mensura crenças e atribuições distorcidas relacionadas à VS em crianças e adolescentes. Composta por 18 itens, respondidos por meio de cinco pontos que variam entre Nunca (0) e Sempre (4), divididos em quatro subescalas: sentimentos de diferença com relação aos pares; diminuição da confiança nas pessoas; auto-atribuição dos eventos negativos; e percepção de uma diminuição da credibilidade dos outros em si. Escores mais altos equivalem a maiores indicadores de sentimento de diferença com relação aos pares e de auto-atribuição por eventos negativos, além de menor percepção de credibilidade e de confiança interpessoal.

Inventário de Depressão Infantil (CDI; Cunha, 2001). Composto por 27 itens de escolha múltipla com três alternativas correspondentes. A criança/adolescente deve escolher a opção que melhor descreve o seu estado nos últimos dias. As opções são pontuadas de zero a dois. $\mathrm{O}$ ponto de corte do CDI foi estabelecido em 19 pontos. $\mathrm{O}$ CDI foi adaptado para uso no Brasil por Gouveia, Barbosa, Almeida e Gaião (1995) demonstrando características psicométricas adequadas para avaliação de sintomas de depressão.

Entrevista Neuropsiquiátrica Internacional MINI para Crianças e Adolescentes (Sheehan, Shytle, Milo, \& Janavs, 2009). Composta de perguntas com possibilidade de respostas dicotômicas (sim e não). Utilizaram-se as entrevistas para investigação de Dependência/Abuso de substância, TDAH, TC e TDO. Foi feito contato com os autores da entrevista, os quais sugeriram a utilização da versão portuguesa (Portugal; Guerreiro, Navarro, Mendes, \& Sampaio, 2009), tendo em vista que não havia uma versão brasileira. A versão portuguesa foi adaptada ao português brasileiro por uma psicóloga de origem portuguesa residente no Brasil e passou pela revisão de outros dois profissionais brasileiros. Os participantes da pesquisa não tiveram dificuldades em respondê-la.

Escala de Estresse Infantil (ESI; Lipp \& Lucarelli, 1998). Composta por 35 itens, cujas respostas são feitas por meio de uma escala de cinco pontos, na qual a criança ou adolescente pinta um círculo dividido em quatro partes, conforme a frequência (Nunca, Um pouco, Às vezes, Quase sempre e Sempre) com que experimentam os sintomas indicados nos itens. Pode-se considerar que a criança/ adolescente avaliada possui indicadores significativos de estresse quando aparecem sete ou mais círculos comple- tamente cheios (pintados) da escala total ou o escore total é maior do que 105 pontos ou, ainda, é igual ou superior a 27 pontos em qualquer uma das três subescalas a seguir: reações físicas, reações psicológicas, reações psicológicas com componente depressivo ou, ainda, quando o escore for igual ou superior a 24 pontos na subescala reações psicofisiológicas.

Inventário de Ansiedade Traço-Estado para Crianças (IDATE-C; Biaggio \& Spielberger, 1983). Constituído de duas escalas, cada uma com 20 itens, do tipo auto-avaliação, que visam a medir indicadores de dois conceitos distintos de ansiedade: traço e estado. Cada item é composto por três afirmações que representam diferentes intensidades do sintoma. Escores mais altos indicam maior presença de ansiedade em ambas as escalas.

Entrevista Estruturada com Base no DSM IV/ SCID (Del Bem et al., 2001, adaptada por Habigzang, 2006). Os critérios diagnósticos estabelecidos pelo Manual Diagnóstico e Estatístico dos Transtornos Mentais (DSM-IV-TR; American Psychiatry Association, 2002) são utilizados como base para identificação da presença dos sintomas de TEPT.

Ficha de Avaliação dos Relatos da Intervenção. Produzida para utilização neste estudo. É composta por duas partes: Na primeira foram avaliados três indicadores intervalares - (a) Aliança terapêutica, subdividido em "Vínculo de confiança" (i.e., participante demonstrou carisma pelo(a) psicoterapeuta, relatou conteúdos importantes e não escondeu informações importantes do(a) psicoterapeuta), "Relação de trabalho" (i.e., o participante realizou as atividades propostas, feedback dado pelo participante em relação ao(à) psicoterapeuta) e "Postura do(a) psicoterapeuta" (i.e., foi atento, ativo, bem humorado, empático, ouviu o participante e o deixou se expressar); (b) Participação nas sessões, subdividido em "Comparecimento" (i.e., presenças às sessões, ausências foram comunicadas com antecedência e/ou justificadas, a(s) faltas(s) foram voluntárias ou involuntárias), "Pontualidade" (i.e., respeito ao horário das sessões, comunicação de atrasos com antecedência, o(s) atraso(s) foram voluntários ou involuntários) e "Auto-revelação (i.e., participante falou sobre o problema e/ou falou sobre demais aspectos importantes - assuntos particulares); e (c) Resposta às técnicas empregadas, subdividido em "Compreensão das técnicas utilizadas"(i.e., participante compreendeu a proposta de realização e engajou-se nas técnicas) e "Vivência das técnicas empregadas" (i.e., participante utilizou as técnicas em seu cotidiano) - por meio de uma escala de cinco pontos que variam de Totalmente ausente $(0)$ à Totalmente presente (5). Na segunda parte, cada sessão do modelo adaptado foi avaliada quanto aos indicadores categóricos "Estrutura" (i.e., as sessões seguiram a estrutura básica), “Objetivos” (i.e., após a realização de cada sessão foi possível perceber que os objetivos propostos foram alcançados) e "Tarefas de casa" (i.e., as tarefas de 
casa propostas foram realizadas pelo participante) por meio de três opções de resposta: Sim (quando o indicador esteve presente), Em parte (quando o indicador esteve parcialmente presente) e $N a ̃ o$ (quando o indicador não esteve presente). A escolha e operacionalização dos indicadores utilizados foi baseada em estudos prévios (Dobson \& Dobson, 2010; Tetley, Jinks, Huband, \& Howells, 2011), bem como na consulta a duas psicólogas experts em TCC com crianças e adolescentes.

\section{Resultados}

\section{Avaliação Inicial e Final}

Os três participantes não apresentaram sintomas de Dependência/Abuso de Álcool, Dependência/Abuso de Substância/Não Álcool e TDO, avaliados por meio da entrevista MINI. Ao analisar os demais escores dos participantes nas avaliações inicial e final, percebe-se que estes diferiram, embora não se possa afirmar se houve diminuição ou aumento significativo no número de sintomas devido à impossibilidade de se realizar análises estatísticas (Tabela 2).

Tabela 2

Escores dos Instrumentos Psicológicos Antes e Após a Intervenção

\begin{tabular}{|c|c|c|c|c|c|c|c|}
\hline \multicolumn{2}{|l|}{ Sintoma (Instrumento) } & \multicolumn{2}{|c|}{ A } & \multicolumn{2}{|c|}{ B } & \multicolumn{2}{|c|}{$\mathrm{C}$} \\
\hline & & Pré-Teste & Pós-Teste & Pré-Teste & Pós-Teste & Pré-Teste & Pós-Teste \\
\hline \multicolumn{2}{|l|}{ Depressão (CDI) } & 10 & 03 & 14 & 11 & 11 & 05 \\
\hline \multicolumn{2}{|l|}{ Estresse (ESI) } & 46 & 14 & 38 & 35 & 35 & 31 \\
\hline \multirow{2}{*}{$\begin{array}{l}\text { Ansiedade } \\
\text { (IDATE - C) }\end{array}$} & Estado & 36 & 38 & 27 & 28 & 30 & 28 \\
\hline & Traço & 39 & 32 & 34 & 34 & 35 & 31 \\
\hline \multirow{3}{*}{$\begin{array}{l}\text { TEPT } \\
\text { (Entrevista Clínica) }\end{array}$} & Revivência & 04 & 00 & 00 & 02 & 00 & 01 \\
\hline & Evitação & 01 & 00 & 01 & 02 & 01 & 02 \\
\hline & Excitabilidade & 01 & 00 & 02 & 02 & 01 & 01 \\
\hline \multirow{4}{*}{$\begin{array}{l}\text { Atribuições } \\
\text { e Percepções } \\
\text { Distorcidas (CAPS) }\end{array}$} & Diferença pares & 07 & 06 & 06 & 07 & 06 & 05 \\
\hline & Sentimentos culpa & 11 & 07 & 08 & 08 & 08 & 04 \\
\hline & Credibilidade & 07 & 08 & 07 & 08 & 05 & 10 \\
\hline & Confiança & 14 & 12 & 07 & 09 & 11 & 07 \\
\hline \multicolumn{2}{|l|}{ TDAH (MINI) } & 05 & 01 & 00 & 00 & 09 & 07 \\
\hline \multicolumn{2}{|l|}{ TC (MINI) } & 04 & 03 & 00 & 00 & 01 & 01 \\
\hline
\end{tabular}

Avaliação da Aplicação do Modelo Adaptado

O modelo adaptado foi avaliado por três juízes independentes a partir dos relatos produzidos após sua aplicação por meio dos indicadores intervalares (i.e., Aliança Terapêutica, Participação nas Sessões e Respostas às Técnicas Empregadas) e categóricos (i.e., Estrutura, Objetivos e Tarefas de Casa) da ficha de avaliação dos relatos da intervenção. A análise de concordância entre juízes para os indicadores intervalares foi realizada por meio do cálculo do Coeficiente de Correlação Intraclasse (CCI; Fleiss, \& Shrout, 1978; McGraw \& Wong, 1996). Na Tabela 3, estão apresentados os resultados dessa análise.

Verificou-se homogeneidade significativa entre as avaliações dos juízes em todos os subitens do indicador Aliança terapêutica: Vínculo de Confiança $(\mathrm{CCI}=0,871$, $p=0,057)$, Relação de Trabalho $(\mathrm{CCI}=0,805, p<0,05)$ e Postura do(a) Psicoterapeuta (CCI $=0,923, p<0,05)$; e no subitem Auto-revelação do indicador Participação nas Sessões ( $98 \%$ de concordância). Nos demais indicadores não houve homogeneidade significativa.

Embora o CCI permita verificar se há homogeneidade entre avaliações de diferentes juízes, ele não permite identificar se a avaliação dos juízes é favorável ou desfavorável aos itens. Assim, foram calculadas as médias e os desvios padrão de cada um dos indicadores e de seus subitens (ver Tabela 4). 
Hohendorff, J. V., Salvador-Silva, R., Andrade, R., Habigzang, L. F. \& Koller, S. H. (2014). Adaptação e Avaliação de uma Intervenção Cognitivo-Comportamental para Meninos Vítimas de Violência Sexual.

Tabela 3

Coeficiente de Correlação Intraclasse dos Oito Indicadores Intervalares de Avaliação da Intervenção

\begin{tabular}{lcc}
\hline Indicador & CCI & $95 \%$ IC \\
\hline Aliança Terapêutica & & \\
$\quad$ Vínculo de Confiança & $0,871^{+}$ & $-0,384-0,997$ \\
$\quad$ Relação de Trabalho & $0,805^{*}$ & $-0,035-0,995$ \\
$\quad$ Postura do(a) Psicoterapeuta & $0,923^{*}$ & $0,440-0,998$ \\
Participação nas Sessões & & \\
Comparecimento & 0,00 & $3,784-0,983$ \\
Pontualidade & 0,500 & $12,096-0,989$ \\
Auto-revelação & $98 \% \%^{\mathrm{a}}$ & - \\
Resposta às Técnicas Empregadas & & $-0,857-0,994$ \\
Compreensão das Técnicas Utilizadas & 0,750 & $11,002-0,983$ \\
$\quad$ Vivência das Técnicas Utilizadas & 0,200 & \\
\hline
\end{tabular}

Nota. $\mathrm{CCI}(3,3)$ com Concordância Absoluta.

${ }^{a}$ Como os juízes 1 e 2 tiveram variância zero nas suas avaliações, calculou-se a frequência de concordância entre os juízes. ${ }^{*} p<0,05,{ }^{\dagger} p=0,057$.

Tabela 4

Médias e Desvios Padrão dos Oito Indicadores Intervalares de Avaliação da Intervenção

\begin{tabular}{lcccc}
\hline Indicador & Participante A & Participante B & Participante C & Total \\
\hline Aliança Terapêutica & & & & \\
$\quad$ Vínculo de Confiança & $3,67(0,58)$ & $5,00(0,00)$ & $3,67(0,58)$ & $4,11(0,77)$ \\
$\quad$ Relação de Trabalho & $3,33(0,58)$ & $5,00(0,00)$ & $4,00(1,00)$ & $4,11(0,84)$ \\
$\quad$ Postura do(a) Psicoterapeuta & $4,00(0,00)$ & $5,00(0,00)$ & $3,67(0,58)$ & $4,22(0,69)$ \\
Total do Indicador & - & - & - & $4,15(0,06)$ \\
Participação nas Sessões & & & & \\
$\quad$ Comparecimento & $4,00(1,00)$ & $4,00(1,00)$ & $5,00(0,00)$ & $4,33(0,58)$ \\
$\quad$ Pontualidade & $3,67(0,58)$ & $4,33(0,58)$ & $4,67(0,58)$ & $4,22(0,51)$ \\
$\quad$ Autorrevelação & $5,00(0,00)$ & $5,00(0,00)$ & $4,67(0,58)$ & $4,89(0,19)$ \\
Total do Indicador & - & - & - & $4,48(0,34)$ \\
Resposta às Técnicas Empregadas & & & & \\
$\quad$ Compreensão das Técnicas Utilizadas & $4,00(1,00)$ & $4,67(0,58)$ & $4,00(1,00)$ & $4,22(0,38)$ \\
$\quad$ Vivência das Técnicas Utilizadas & $3,67(0,58)$ & $4,67(0,58)$ & $3,67(1,15)$ & $4,00(0,58)$ \\
Total do Indicador & - & - & - & $4,11(0,15)$ \\
\hline
\end{tabular}


A análise das médias para cada um dos indicadores revelou que elas foram próximas ao escore máximo possível na escala de cinco pontos $(0=$ Totalmente ausente a $5=$ Totalmente presente). $\mathrm{O}$ indicador Participação nas Sessões foi o que obteve maior média $(M=4,48, D P=$ $0,34)$, seguido dos indicadores Aliança Terapêutica $(M=$ $4,15, D P=0,06)$, e Resposta às Técnicas Empregadas $(M$ $=4,11, D P=0,15$ ).

A análise dos indicadores Estrutura, Objetivos e Tarefas de Casa foi realizada por meio do cálculo da porcentagem de concordância entre os três juízes. Optou-se por tal análise devido ao tipo de variável (categórica - "Sim", "Parcialmente", e "Não") e da pouca variabilidade entre as avaliações - fato que pode estar relacionado ao reduzido número de casos avaliados (i.e., três). A porcentagem de concordância entre juízes para cada indicador em cada sessão foi calculada por meio do somatório do total de vezes em que houve concordância entre as avaliações, considerando o número máximo de vezes em que os juízes poderiam concordar (nove - três juízes x três participantes). As porcentagens de concordância para cada sessão em cada um dos indicadores estão apresentadas na Tabela 5.

Tabela 5

Porcentagem de Concordância dos Três Indicadores Categóricos de Avaliação da Intervenção

\begin{tabular}{lcccccccccccccccc}
\hline & \multicolumn{11}{c}{ Concordância em cada sessão (\%) } \\
\cline { 2 - 6 } & 1 & 2 & 3 & 4 & 5 & 6 & 7 & 8 & 9 & 10 & 11 & 12 & 13 & 14 & 15 & 16 \\
\hline Estrutura & 78 & 89 & 89 & 89 & 89 & 100 & 89 & 100 & 89 & 89 & 89 & 100 & 78 & 100 & 100 & 100 \\
Objetivos & 89 & 67 & 67 & 78 & 89 & 44 & 89 & 89 & 67 & 89 & 89 & 100 & 89 & 89 & 100 & 89 \\
Tarefas de casa & - & - & - & - & 44 & 78 & - & - & - & - & - & - & - & - & - & - \\
\hline
\end{tabular}

O indicador Estrutura, no qual foi avaliado se o(a) psicoterapeuta seguiu a estrutura de uma sessão TCC, foi o que obteve as maiores porcentagens, variando de $78 \%$ a 100\%, de concordância nas avaliações. Já o indicador Objetivos, no qual foi avaliado se ao final de cada sessão foi possível observar que os objetivos propostos de acordo com o modelo adaptado foram alcançados, obteve concordâncias entre $44 \%$ e $100 \%$. O indicador Tarefas de Casa, no qual foi avaliado se as tarefas de casa propostas foram realizadas pelos participantes, obteve as menores porcentagens de concordância (44\% a 78\%).

Após analisar a porcentagem de concordância entre os juízes, buscou-se verificar se as avaliações dos juízes foram favoráveis ("Sim", "Parcialmente") ou desfavoráveis ("Não") a cada indicador analisado. Tal análise foi realizada com o objetivo de complementar a análise das porcentagens de concordância por meio da construção de gráficos de frequência de respostas para cada sessão em cada indicador (Figura 1).

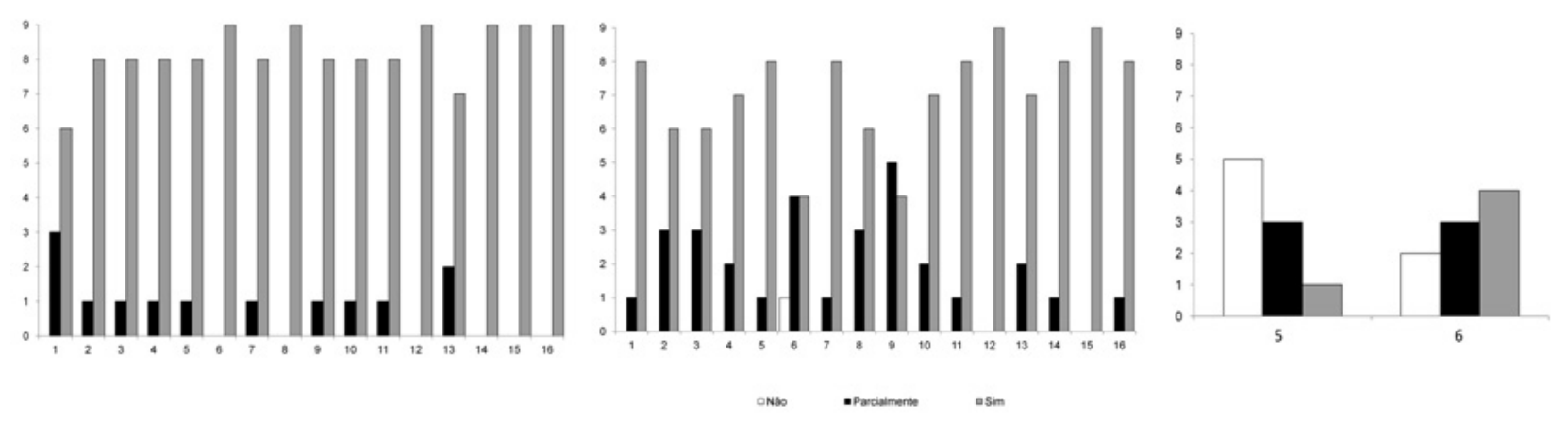

Figura 1. Frequência de respostas dos indicadores categóricos estrutura, objetivos e tarefas de casa.

A análise do indicador Estrutura revelou que todas as dezesseis sessões obtiveram número superior de avaliações "Sim", sendo que as sessões seis, oito, 12, 14, 15 e 16 obtiveram somente respostas "Sim". O indicador Objetivos revelou que somente as sessões seis e nove não obtiveram número superior de avaliações "Sim", enquanto as sessões
12 e 15 obtiveram somente respostas "Sim", ou seja, os três juízes concordaram plenamente (100\%) que os objetivos foram alcançados. A análise do indicador Tarefas de Casa foi realizada somente para as sessões cinco e seis, pois somente essas possuíam tarefas de casa. Os resultados obtidos revelam que houve predomínio de respostas "Não" 
Hohendorff, J. V., Salvador-Silva, R., Andrade, R., Habigzang, L. F. \& Koller, S. H. (2014). Adaptação e Avaliação de uma Intervenção Cognitivo-Comportamental para Meninos Vítimas de Violência Sexual.

(5) na sessão cinco, além de respostas "Parcialmente" (3) e "Sim" (1). A porcentagem de concordância para as avaliações dessa sessão foram de $89 \%$. Com relação à sessão seis, os resultados indicaram que houve predomínio de respostas "Sim" (4), seguido de repostas "Parcialmente" (3) e "Não" (2), com porcentagem de concordância de 44\%.

\section{Discussão}

O objetivo deste estudo foi o de adaptar, aplicar e avaliar um modelo de intervenção cognitivo-comportamental para meninos vítimas de VS. Especificamente em relação à VS contra crianças e adolescentes, destaca-se a importância de se investigar sua ocorrência e alternativas de tratamento para vítimas masculinas, tendo em vista a escassez de estudos sobre essa temática.

A análise dos escores obtidos pelos três participantes nas avaliações revelaram diferentes repostas à intervenção em relação aos sintomas investigados. Ao se comparar os escores, somente o número de sintomas de depressão e estresse foi menor para os três participantes após a aplicação do modelo adaptado. Os escores dos demais sintomas apresentaram variabilidade dentre os participantes. Tais resultados foram semelhantes àqueles encontrados no estudo de Grayston e De Luca (1995). Diversos fatores podem ter influenciado essa variabilidade, dentre eles destaca-se a história de vida de cada menino. Por exemplo, fatores como a duração da VS, relacionamento com o irmão/agressor e reação da mãe podem ter influenciado a resposta do participante $\mathrm{B}$, que apresentou maior número de sintomas após a intervenção. Tais fatores são indicados pela literatura como mediadores do impacto da VS (Araújo, 2002; Furniss, 1993; Kristensen, 1996; Sanderson, 2005). Demais fatores, tais como o cometimento de VS contra o irmão, a situação de acolhimento institucional (participante A), contato com o agressor (participante C) podem estar relacionados aos sintomas de ansiedade, TC e percepção de menor credibilidade. Além disso, fatores vinculados à intervenção em si, tais como o número de sessões para utilização de técnicas de exposição gradual às memórias traumáticas, podem estar relacionados ao aumento dos sintomas de TEPT verificado.

Apesar das evidências consistentes acerca da efetividade de técnicas de exposição gradual às memórias traumáticas na redução de sintomas de TEPT, sabe-se que ela pode não beneficiar todas as crianças ou adolescentes devido à heterogeneidade na apresentação do TEPT nessas etapas do desenvolvimento (Bryant, 2000). Em alguns casos, devido à intensidade da ansiedade mobilizada com técnicas de exposição gradual às memórias traumáticas, pode ser necessário maior número de sessões (Cohen, 2003). O número de sessões de exposição à memória traumática é uma importante variável que vem sendo estudada com o intuito de verificar sua possível relação com os resultados de intervenções cognitivo-comportamentais. Em estudo recente, crianças e adolescentes que receberam tratamento com 16 sessões apresentaram menos sintomas de reexperiência da memória traumática e evitação, quando comparados aqueles que receberam tratamento com oito sessões (Deblinger et al., 2011).

$\mathrm{Na}$ avaliação do modelo de grupoterapia para meninas vítimas de VS (Habigzang et al., 2009) houve redução significativa na quantidade de sintomas de TEPT nas três categorias avaliadas - revivência, evitação e hipervigilância. As técnicas de exposição gradual às memórias traumáticas foram citadas como contribuições significativas para a redução dos sintomas de revivência e evitação. Diante disso, percebe-se a diferença entre as respostas às técnicas de exposição gradual à memória traumática obtidas pelas meninas avaliadas no estudo de Habigzang et al. (2009) e os meninos participantes deste estudo. Embora sua comparação esteja limitada pelas diferenças entre as modalidades terapêuticas utilizadas (grupal x individual), seus resultados podem indicar que meninos vítimas de VS necessitam número maior de sessões destinadas ao trabalho com a memória traumática em um formato individual de tratamento. Tendo em vista que as técnicas de exposição gradual às memórias traumáticas consistem na recordação e no relato acerca da VS, meninos vítimas podem se sentir mais constrangidos em fazê-lo. Diante disso, o aumento do número de sessões destinadas às técnicas de exposição gradual às memórias traumáticas se constitui em um aspecto a ser modificado no modelo adaptado para utilização com meninos vítimas no formato individual. Nessas sessões, poder-se-ia investir no preparo para as técnicas de exposição gradual às memórias traumáticas por meio da abordagem dos sentimentos de vergonha, estigmatização e medo relacionados à VS.

$\mathrm{O}$ aumento no número de alguns sintomas após a intervenção pode estar vinculado, ainda, a uma possível inibição desses sintomas no período pré-intervenção. Por meio de entrevistas com 26 homens vítimas de VS (idade média de 33,7 anos) na infância, foi identificado que os participantes tenderam a inibir o processo psicológico que comumente ocorre após a VS, ou seja, expressão de sentimentos, como tristeza, medo e abandono. Uma possível explicação fornecida pelo autor para essa inibição reside no fato de que a vitimização masculina é percebida como antagônica aos estereótipos de masculinidade (i.e., ser forte, ativo, e estar no controle das situações). Diante disso, os participantes podem não ter demonstrado seus sofrimentos, uma vez que isso não condiz com o que se espera socialmente de um homem (Lisak, 1994).

A participação na intervenção pode ter propiciado um ambiente de acolhimento e aceitação no qual os meninos perceberam que poderiam expressar seus sofrimentos, permitindo-se experienciar o processo psicológico decorrente da VS. Diante disso, as intervenções psicoterapêuticas com meninos vítimas de VS talvez necessitem de um número maior de sessões, nas quais se busque sensibilizar os participantes para os sintomas que podem estar sendo inibidos e, após, intervir nesses aspectos. 
Em relação à avaliação da aplicação do modelo adaptado, realizada por meio da ficha de avaliação dos relatos da intervenção, obteve-se adequação quanto ao indicador Aliança Terapêutica e o subitem Autorrevelação do indicador Participação nas Sessões, além dos indicadores Estrutura e Objetivos.

Esses resultados, tomados em conjunto, podem indicar que a formação da aliança terapêutica contribuiu para que os participantes se autorrevelassem (Falcone, 2011), ou seja, falassem sobre o problema (VS) e a respeito de assuntos particulares, tais como dificuldades em apresentar trabalhos na sala de aula. Ao se trabalhar com crianças e adolescentes vítimas de VS, deve-se estar atento para o fato de que a maior parte das vitimizações ocorre no meio intrafamiliar (Habigzang, 2010; Sanderson, 2005), configurando-se assim, como uma situação na qual vínculos de confiança foram maculados. Devido a isso, a formação da aliança terapêutica, na qual se estabelece um bom vínculo de confiança, é considerada um desafio. Deve-se atentar, no planejamento de intervenções, para o estabelecimento do vínculo de confiança, a postura do psicoterapeuta - se é atento, ativo, bem humorado, empático -, e a relação de trabalho entre esse e o paciente.

Em contrapartida, a avaliação dos subitens Comparecimento e Pontualidade do indicador Participação nas sessões, e os indicadores Resposta às Técnicas Empregadas e Tarefas de Casa não foram favoráveis. Aspectos como atrasos e faltas dos participantes às sessões, embora por motivos involuntários (problemas de transporte, negligência dos cuidadores, por exemplo) e a não realização das tarefas de casa e a recusa inicial dos participantes em realizar as atividades do TIE podem ter influenciado o resultado desses indicadores.

Os resultados deste estudo se constituem, então, como evidências iniciais da adequação do modelo adaptado para o tratamento dos participantes. Tais resultados podem contribuir com a prática de profissionais da rede de proteção e de atendimento às crianças e adolescentes vítimas de VS, tendo em vista a percepção de falta de preparo técnico que estes possuem em relação ao atendimento dessa população (Conselho Federal de Psicologia, 2009). No entanto, estudos futuros podem refinar o modelo adaptado e contornar as limitações deste estudo, como por exemplo, a utilização do instrumento para a avaliação de transtornos externalizantes (Sheehan et al., 2009). Além de se configurar como um instrumento de autorrelato, o método de tradução desse instrumento incluiu apenas a tradução direta do idioma de origem para o português brasileiro, sem a realização de uma retrotradução. Ademais, não houve um estudo piloto para verificar a adequação do instrumento. Em futuras aplicações do modelo adaptado será necessário, então, incluir um instrumento que possua evidências de validade para a população brasileira. Além disso, foi necessário incluir um participante (A) com histórico de autoria de VS, além da vitimização, o que pode ter enviesado os resultados. Porém, esse aspecto pode ser considerado, também, como uma implicação desse estudo para futuras pesquisas, uma vez que, ao se planejar e avaliar intervenções para meninos vítimas de VS, deve-se cogitar a possibilidade de que vítimas encaminhadas para tratamento possam ter histórico de autoria de VS.

\section{Referências}

Alaggia, R. (2005). Discloing the trauma of child sexual abuse: A gender analysis. Journal of Loss and Trauma, 10, 453-470.

American Psychiatry Association. (2002). DSM-IV-TR: Manual diagnóstico e estatístico de transtornos mentais (4. ed. rev., C. Dornelles, Trad.). Porto Alegre, RS: Artes Médicas.

Araújo, M. F. (2002). Violência e abuso sexual na família. Psicologia em Estudo (Maringá), 7(2), 3-11.

Biaggio, A., \& Spielberger, C. D. (1983). Inventário de ansiedade traço-estado-Idate-C Manual. Rio de Janeiro, RJ: Centro de Psicologia Aplicada.

Bryant, R. A. (2000). Cognitive behavioral therapy of violencerelated posttraumatic stress disorder. Aggression and Violent Behavior, 5, 79-97.

Cohen, J. A. (2003). Treating acute posttraumatic reactions in children and adolescents. Society of Biologial Psychiatry, 53, 827-833.

Conselho Federal de Psicologia. (2009). Serviço de proteção social a crianças e adolescentes vítimas de violência, abuso e exploração sexual e suas famílias: Referências para a atuação do psicólogo. Brasília, DF: Autor.

Cunha, J. A. (2001). Manual da versão em português das Escalas Beck. São Paulo, SP: Casa do Psicólogo.

Deblinger, E., Mannarino, A. P., Cohen, J. A., Runyon, M. K., \& Steer, R. A. (2011). Trauma-focused cognitive behavioral therapy for children: Impact of the trauma narrative and treatment length. Depression and Anxiety, 28, 67-75.

Del Ben, C. M., Vilela, J. A., Crippa, J. A., Hallak, J. E., Labate, C. M., \& Zuardi, A. W. (2001). Confiabilidade da entrevista estruturada para o DSM-IV - Versão clínica traduzida para o português. Revista Brasileira de Psiquiatria, 23, 156-159.

Dobson, D., \& Dobson, K. S. (2010). A terapia cognitivo-comportamental baseada em evidências (V. D. Figueira, Trad.). Porto Alegre, RS: Artmed.

Falcone, E. M. O. (2011). Relação terapêutica como ingrediente ativo de mudança. In B. Rangé (Ed.), Psicoterapias cognitivo-comportamentais: Um diálogo com a psiquiatria (pp. 145154). Porto Alegre, RS: Artmed.

Fleiss, J. L., \& Shrout, P. E. (1978). Approximate interval estimation for a certain intraclass coefficient. Psychometrika, 43, 259-262.

Furniss, T. (1993). Abuso sexual da criança: Uma abordagem multidisciplinar (M. A. V. Veronese, Trad.). Porto Alegre, RS: Artes Médicas.

Gouveia, V. V., Barbosa, G. A., Almeida, H. J. F., \& Gaião, A. A. (1995). Inventário de Depressão Infantil - CDI: Estudo de adaptação com escolares de João Pessoa. Jornal Brasileiro de Psiquiatria, 44, 345-349.

Grayston, A. D., \& De Luca, R. (1995). Group therapy for boys who have experienced sexual abuse: Is it the treatment of choice? Journal of Child and Adolescent Group Therapy, 5(2), 57-82.

Guerreiro, D. F., Navarro, R., Mendes, R., \& Sampaio, D. (2009). Entrevista neuropsiquiátrica internacional MINI para crianças e adolescentes - Versão Portuguesa 6.0. Lisboa, Portugal: Departamento de Psiquiatria e Saúde Mental, Hospital de Santa Maria. 
Hohendorff, J. V., Salvador-Silva, R., Andrade, R., Habigzang, L. F. \& Koller, S. H. (2014). Adaptação e Avaliação de uma Intervenção Cognitivo-Comportamental para Meninos Vítimas de Violência Sexual.

Habigzang, L. F. (2006). Avaliação e intervenção clínica para meninas vitimas de abuso sexual intrafamiliar (Dissertação de mestrado, Instituto de Psicologia, Universidade Federal do Rio Grande do Sul, Porto Alegre, RS, Brasil).

Habigzang, L. F. (2010). Avaliação de impacto e processo de um modelo de grupoterapia cognitivo-comportamental para meninas vítimas de abuso sexual (Tese de doutorado, Instituto de Psicologia, Universidade Federal do Rio Grande do Sul, Porto Alegre, RS, Brasil).

Habigzang, L. F., Damásio, B. F., Hohendorff, J. V., \& Koller, S. H. (2011). Intersections between scientific research and public policies for sexual abuse. International Journal of Behavioural Development Bulletin, 35(6), 15-18.

Habigzang, L. F., Stroeher, F. H., Hatzenberger, R., Cunha, R. C., Ramos, M. S., \& Koller, S. H. (2009). Grupoterapia cognitivo-comportamental para crianças e adolescentes vítimas de abuso sexual. Revista de Saúde Pública, 43, 70-78.

Hetzel-Riggin, M., Brausch, A. M., \& Montgomery, B. S. (2007). A meta-analytic investigation of therapy modality outcomes for sexually abused children and adolescents: An exploratory study. Child Abuse \& Neglect, 31, 125-141.

Hohendorff, J. V., Bavaresco, P. D., Habigzang, L. F., \& Koller, S. H. (2012). Abuso sexual contra meninos: Uma revisão. In L. F. Habigzang \& S. H. Koller (Eds.), Violência contra crianças e adolescentes: Teoria, pesquisa e prática (pp. 105122). Porto Alegre, RS: Artmed.

Hohendorff, J. V., Habigzang, L. F., \& Koller, S. H. (2013). Descrição e processo de adaptação de um modelo de terapia cognitivo-comportamental para meninos vítimas de violência sexual. In A. P. Serafim, D. M. Barros, \& F. Saffi (Eds.), Temas em psiquiatria forense e psicologia jurídica III: Violência sexual (Vol. 3, pp. 249-270). São Paulo, SP: Vetor.

Hohendorff, J. V., Habigzang, L. F., \& Koller, S. H. (2012). Violência sexual contra meninos: Dados epidemiológicos, características e consequências. Psicologia USP, 23, 395-415.

Hohendorff, J. V., Habigzang, L. F., Rodrigues, L. H., \& Koller, S. H. (2012). Produção e utilização de um documentário sobre violência sexual contra meninos. Psico, 43, 228-236.

Holmes, W. C., \& Slap, G. B. (1998). Sexual abuse of boys: Definition, prevalence, correlates, sequelae and management Journal of American Medical Association, 180, 1855-1862.

Kristensen, C. H. (1996). Abuso sexual em meninos (Dissertação de mestrado, Instituto de Filosofia e Ciências Humanas, Universidade Federal do Rio Grande do Sul, Porto Alegre, RS, Brasil)

Lipp, M. E., \& Lucarelli, M. D. M. (1998). Escala de Stress Infantil-ESI: Manual. São Paulo, SP: Casa do Psicólogo.

Lisak, D. (1994). The psychological impact of sexual abuse: Content analysis of interviews with male survivors. Journal of Traumatic Stress, 7, 525-548.

Lucânia, E. L., Valério, N. I., Barison, S. Z. P., \& Miyazaki, M. C. O. S. (2009). Intervenção cognitivo-comportamental em violência sexual: Estudo de caso. Psicologia em Estudo (Maringá), 14, 817-826.

Maikovich-Fong, A. K., \& Jaffee, S. R. (2010). Sex differences in childhood sexual abuse characteristics and victims' emotional and behavioral problems: Findings from a national sample of youth. Child Abuse \& Neglect, 34, 429-437.

Maniglio, R. (2009). The impact of child sexual abuse on health: A systematic review of reviews. Clinical Psychology Review, 29, 647-657.
Mannarino, A. P., Cohen, J. A., \& Berman, S. R. (1994). The children's attributions and perceptions scale: A new measure of sexual abuse-related factors. Journal of Clinical Child Psychology, 23, 204-211.

McGraw, K. O., \& Wong, S. P. (1996). Forming inferences about some intraclass correlation coefficients. Psychological Methods, 1, 30-46.

O'Leary, P. J. O. (2009). Men who were sexually abused in childhood: Coping strategies and comparisons in psychological functioning. Child Abuse \& Neglect, 33, 471-479.

Pinto, A. A., Jr. (2005). Violência sexual doméstica contra meninos: Um estudo fenomenológico. São Paulo, SP: Vetor.

Pires, M. F., Filho. (2007). Violência intrafamiliar: A compreensão de psicólogos que atendem em instituições crianças do sexo masculino, vítimas de abuso sexual (Dissertação de mestrado, Curso de Mestrado em Psicologia Clínica, Universidade Católica de Pernambuco, Recife, PE, Brasil).

Sanderson, C. (2005). Abuso sexual em crianças: Fortalecendo pais e professores para proteger crianças contra abusos sexuais e pedofilia (F. de Oliveira, Trad). São Paulo, SP: M. Books do Brasil.

Sheehan, D., Shytle, D., Milo, K., \& Janavs, J. (2009). Entrevista neuropsiquiátrica internacional MINI para crianças e adolescentes. Tampa, FL: University of South Florida College of Medicine.

Sigurdardottir, S., Halldorsdottir, S., \& Bender, S. S. (2012). Deep and almost unbearable suffering: Consequences of childhood sexual abuse for men's health and well-being. Scandinavian Journal of Caring Sciences, 26, 688-697.

Tetley, A., Jinks, M., Huband, N., \& Howells, K. (2011). A systematic review of measures of therapeutic engagement in psychosocial and psychological treatment. Journal of Clinical Psychology, 67, 927-941.

The Metropolitan Toronto Special Committee on Child Abuse. (1995). Child Sexual Abuse Protocol (3 ${ }^{\text {rd }}$ ed.). Toronto, Canada: Author.

Weiss, K. G. (2010). Male sexual victimization: Examining men's experiences of rape and sexual assault. Men and Masculinities, 12, 275-298.

Yin, R. K. (2005). Estudo de caso: Planejamento e métodos (D. Grassi, Trad.). Porto Alegre, RS: Bookman. (Original publicado em 2003) 